\title{
Radical Scavenging Activity of Tertiary Butyl Hydroquinone and Assessment of Stability of Palm Oil (Elaeis guineensis) by Ultrasonic Studies
}

\author{
M. Banu, ${ }^{\mathrm{a}, *}$ Siddaramaiah, ${ }^{\mathrm{b}}$ and N. Prasad \\ ${ }^{a}$ Department of Chemistry, Vidya Vikas Institute of Engineering \\ \& Technology, Mysore, Karnataka, 570028, India \\ bDepartment of Polymer Science \& Technology, \\ Sri Jayachamarajendra College of Engineering, \\ Mysore, Karnataka, 570 006, India \\ 'Department of Chemistry, Govt Engineering College, \\ Chamarajanagar, Karnataka, India
}

doi: 10.15255/CABEQ.2015.2185

Original scientific paper

Received: January 27, 2015

Accepted: December 9, 2016

\begin{abstract}
The aim of the current investigation is to evaluate the efficiency of tertiary butyl hydroquinone (TBHQ) as an antioxidant in edible oil like palm oil (Elaeis guineensis) by physical, chemical and ultrasonic properties. The effects of the varying amounts of TBHQ on the oxidation stability of palm oil have been investigated. The antioxidant incorporated palm oil system and control oil were subjected to heating at $180 \pm 5^{\circ} \mathrm{C}$ continuously for a period of $4 \mathrm{~h}$ per day for 4 days consecutively. The parameters used to assess the thermal degradation and oxidation properties of the palm oil include ultrasonic velocity, viscosity and density. Adiabatic compressibility, intermolecular free length, relaxation time, and acoustic impedance have been calculated from experimental data. The effect of thermal ageing on the physical properties of the oil was confirmed by chemical analysis, which included free fatty acid, total polar compounds, and estimation of fatty acids profile by gas chromatography (GC). The chemical changes were studied by FT-IR bands. The results obtained from ultrasonic studies have shown improvement in oxidative stability and retardation in thermal degradation of the antioxidant-loaded oil in comparison with the base oil. Hence, the ultrasonic velocity and acoustical parameters used for assessment of stability of frying oil and palm oil with TBHQ can be recommended for repeated frying without adverse effects on the physical properties.
\end{abstract}

Key words:

palm oil, TBHQ, ultrasonic velocity, acoustical parameters, FT-IR, gas chromatography

\section{Introduction}

Heat treatment is a widely used process in food processing industries. As heat-processed foods are much appreciated, analytical studies that can assess the changes caused by heating have been demanded from researchers ${ }^{1-4}$. Oils and fats, when heated, suffer thermal oxidation and produce compounds such as peroxides. The peroxides turn into aldehydes, ketones, epoxides, dimers and polymers, undermining the quality of food ${ }^{5}$, while the physical properties of the oils and fats are changed ${ }^{6}$. In order to minimize such effects, the food industry makes use of antioxidants ${ }^{7}$. The important antioxidants used in the food industries are butylated hydroxyanisole (BHA), butyl-1-4-hydroxytoluene (BHT), tert-butyl hydroquinone (TBHQ), and propylgallate (PG), of which TBHQ has been found to be the most effective antioxidant $^{8,9}$.

"Corresponding author: E-mail: mujeedabanu@gmail.com
According to USDA code of Federal Regulations, 'antioxidants' are substances used to preserve fats, oils, and foods by retarding deterioration, rancidity or discolouration due to oxidation. Edible oils with unsaturated fatty acids, especially polyunsaturated fatty acids, are more susceptible to oxidation $^{10,11}$. The use of antioxidants is the most preferred way to inhibit lipid oxidation ${ }^{12}$ as the addition of polyphenols to foods and biological systems scavenge free radicals ${ }^{13-15}$.

The activity of an antioxidant can be estimated by quantitative estimation of primary or secondary products of autoxidationof fats ${ }^{16,17}$, oils, and foods or by monitoring other variables. The primary products such as hydro peroxides formed during lipid oxidation are measured by iodometric titration, which is generally expressed as the peroxide value $(\mathrm{PV})^{18,19}$. The secondary products of oxidation occur when the hydroperoxide decompose to form carbonyl and other compounds. Of the available methods for measuring hydroperoxides decomposition 
products, determination of aldehydic compounds (Anisdine value-AV, Thiobarbituric acid value-TBA $)^{20}$, measurement of total carbonyls ${ }^{21}$ or selected carbonyl compounds and assessment of off-flavours and off-odours due to the formation of volatile decomposition products of hydroperoxides by objective and subjective means are used extensively. Since these methods are time consuming and laborious, they cannot be employed for accepting or rejecting the products. Most sophisticated methods, such as nuclear magnetic resonance (NMR), near infra-red (NIR) spectroscopy, and mass spectra (MS) etc., ${ }^{22,23,54}$ meet the requirements but are cost intensive. Therefore, a cost effective and simple analytical method needs to be developed to meet the above requirements.

Ultrasound represents an innovative, and emerging technology as a destructive or non-destructive one. It has a frequency range of 16-100 $\mathrm{kHz}$ (Power Ultrasonics) followed by a range from $100 \mathrm{kHz}-2 \mathrm{MHz}$ representing the sonochemistry range, and at the end the non-destructive range from $2 \mathrm{MHz}$ up to $10 \mathrm{MHz}^{25-27}$. Ultrasonic methods are simple, instantaneous, and cost-effective for the study of the physical characteristics of various oil products for quality control purposes ${ }^{28}$. Although limited information is available on the determination of physical properties of triglycerides by using the ultrasonic interferometry technique ${ }^{29}$, there is scarcity on its application in studying the molecular interaction between lipid and antioxidants.

A few studies have revealed that the measurement of viscosity and density can be used to determine the oxidative stability ${ }^{30-32}$, while ultrasonic velocity is used to understand the molecular interactions ${ }^{29,33,34}$ in the oil. However, the effect of a synthetic antioxidant on the oils has not been fully elucidated by ultrasonic velocity.

Palm oil (PO) with its inherent frying properties is used due to its techno-economic advantages over other oils and fats. Past studies have demonstrated the frying performance of palm olein during continuous frying of snack foods ${ }^{34}$. Palm oil contains saturated fatty acids like palmitic acid (44.3 $\%)$, oleic acid (38.4\%) and linoleic acid (10.8\%), vitamin $\mathrm{E}$ especially tocotrienols, vitamin $\mathrm{K}$ and magnesium. The antioxidant activity of palm oil is due to the presence of carotenoids and vitamin $\mathrm{E}$. Beta carotene is the reason for the yellow colour of the PO, it may also be an important factor for the free radical scavenging activity. However, palm oil is not very stable at high temperatures and deteriorates on repeated heating and/or frying.

The objectives of this research were: (i) to investigate the TBHQ activity in palm oil using ultrasonic velocity, density, and viscosity; (ii) to study the secondary parameters such as adiabatic com- pressibility, intermolecular free length, relaxation time, acoustic impedance, and (iii) fatty acid profile by gas chromatography (GC), total polar compounds (TPC), free fatty acid (FFA), and spectral changes in FT-IR to correlate the chemical changes in $\mathrm{PO}$ and antioxidant activity.

\section{Experimental study}

\section{Materials}

Fresh palm oil (PO) was obtained from Pentagon Overseas India Limited, India. TBHQ was purchased from Sigma Aldrich, and AR grade chemicals were used for the chemical analysis. Samples of palm oil (control) and palm oil with 50, 100, 150 and $200 \mathrm{ppm}$ of TBHQ were heated in an oil bath at a temperature of $180{ }^{\circ} \mathrm{C}$ continuously for $4 \mathrm{~h}$ per day for 4 days. As per ISO 9001:2008 norms (Indian Standard Quality Management System requirements), $150 \mathrm{ppm}$ of TBHQ is allowed in vegetable oils; however, the general standard for food additives allows $200 \mathrm{ppm}(\text { Codex 1995) })^{50}$. Hence, a maximum of $200 \mathrm{ppm}$ TBHQ has been used in this investigation. All measurements of heat-treated oils were carried out in triplicate and average values are reported.

\section{Techniques}

The density $(\rho)$ of the pure oil and its mixtures was measured using a $10 \mathrm{~mL}$ specific gravity bottle, and distilled water was used as reference. The viscosity $(\eta)$ of the oil and oil-antioxidant mixtures were measured using an Ostwald's viscometer immersed in a temperature controlled water bath (Advance Technocracy Inc.; Ambala; India).

The ultrasonic velocity $(U)$ of $\mathrm{PO}$ with and without TBHQ were measured using an ultrasonic interferometer (Mittal Enterprises; New Delhi, India), at $2 \mathrm{MHz}$ frequency with a tolerance of $\pm 0.005 \%$. The accuracy of the instrument is $0.1 \mathrm{~m} \mathrm{~s}^{-1}$.

The adiabatic compressibility $(\beta)$ is defined as the decrease of volume per increase of pressure when no heat flows in or out. Such a change is related to the compressibility of the medium by following the thermodynamic relation;

$$
\beta=\frac{1}{V}\left[\frac{\delta_{\mathrm{v}}}{\delta_{\mathrm{p}}}\right]
$$

where, $V$ is the volume, $\delta_{\mathrm{v}}$ is the relative change in volume, and $\delta_{p}$ is the relative change in pressure. It can also be calculated from the ultrasonic velocity $(U)$ and the density $(\rho)$ of the oil using Newton Laplace $^{35}$ equation as follows; 


$$
\beta=\frac{1}{U^{2} \rho}
$$

The adiabatic compressibility of liquid can be expressed in terms of intermolecular free length $\left(L_{f}\right)$, which is the distance between the surfaces of the neighbouring molecules and is given by the following equation;

$$
L_{f}=K_{T} \beta_{a d}^{\frac{1}{2}}
$$

where, $K_{T}$ is the temperature-dependent constant, i.e. $201.1209 \cdot 10^{-8}$ at $303 \mathrm{~K}$.

The relaxation time is the time taken for the excitation energy to appear as transitional energy, and it depends on the temperature and impurities. The dispersion of the ultrasonic velocity in a mixture reveals information about the characteristic time of the relaxation process that causes dispersion. The relaxation time $(\tau)$ can be calculated using the following equation; ${ }^{29}$

$$
\tau=\frac{4}{3} \beta \eta
$$

The specific acoustic impedance $(Z)$ is as follows: ${ }^{35}$

$$
Z=U \rho
$$

where, $U$ and $\rho$ are the velocity and density of the oil, respectively.

\section{Fatty acid by gas chromatography}

Fatty acid methyl esters (FAME) of the oils were prepared by transesterification, according to AOCS method No: Ce 1-62, 1998 ${ }^{37}$. FAMEs were analyzed by gas chromatography (GC) (Fisons 8000 , Co., Italy), equipped with a hydrogen flame ionization detector (FID) and a fused silica capillary column $(100 \mathrm{~m} \times 0.25 \mathrm{~mm}$ i.d. $)$, coated with 0.20 $\mu \mathrm{m}$ SP2560 (Supelco Inc., Bellefonte, PA) as the stationary phase. The oven temperature was programmed from 140 to $240{ }^{\circ} \mathrm{C}$ at $4 \mathrm{~min}^{-1}$ with an initial hold at $140{ }^{\circ} \mathrm{C}$ for $5 \mathrm{~min}$. The injector and FID were at $260{ }^{\circ} \mathrm{C}$. A reference standard FAME mix (Supelco Inc.) was analyzed under the same operating conditions to determine the peak identity. The FAMEs were expressed as relative area percentage.

The free fatty acid (FFA) content as the percentage of oleic acid was determined using AFNOR NF T 60- 204 method.

The AOCS method Cd 20-91 was used to determine total polar compounds (TPC) of oil. A chromatogram column (21 $\mathrm{mm}$ i.d. and $450 \mathrm{~mm}$ long) was filled with about $30 \mathrm{~mL}$ of a mixture of light petroleum and diethyl ether $(87: 13, \mathrm{v} / \mathrm{v})$. A wad of glass wool was introduced at the lower end of the column with the aid of a glass rod. Slurry of $24 \mathrm{~g}$ of silica gel in about $80 \mathrm{~mL}$ of the solvent mixture was poured into the column. The elution solvent was drained through the column until its level was 10 $\mathrm{cm}$ above the silica gel level. About $4 \mathrm{~g}$ of sea sand was added and the supernatant was drained down to the sand layer. In order to estimate TPC, $2.5 \mathrm{~g}$ of oil was dissolved in $20 \mathrm{~mL}$ of solvent mixture containing light petroleum and diethyl ether $(87: 13 \mathrm{v} / \mathrm{v})$ at room temperature. The volume was then made up to $50 \mathrm{~mL}$ and the resulting solution was introduced into the column and drained off to the level of the sand layer. The non-polar compounds were eluted with $150 \mathrm{~mL}$ of solvent mixture at a flow rate of 2.5 $\mathrm{mL} \mathrm{min}^{-1}$. TPC was calculated using the following equation;

$$
\operatorname{TPC}(\%)=\left[m-m_{1}\right] / 100
$$

where, $m_{1}$ is mass $(\mathrm{g})$ of the non-polar fraction, and $m$ is mass $(\mathrm{g})$ of the sample containing $20 \mathrm{~mL}$ of solution added to the column.

\section{Results and discussion}

In this study, the effects of different heating times and different amounts of TBHQ addition on the thermal oxidative stability of palm oil has been investigated using parameters such as viscosity ${ }^{27,33}$, density, and ultrasonic velocity ${ }^{34,35}$. The study of thermal degradation and stability of the palm oil with antioxidant was carried out by heating the oil at $180 \pm 5{ }^{\circ} \mathrm{C}$ for the desired time. The viscosity, density, and ultrasonic velocity of $\mathrm{PO}$ and $\mathrm{PO}$ with different concentrations of TBHQ were measured at $30{ }^{\circ} \mathrm{C}$.

The effects of different heating time and TBHQ content on density $(\rho)$ of PO are shown in Fig. 1. The density of heated palm oil gradually and substantially increased with heating time. Incorporation

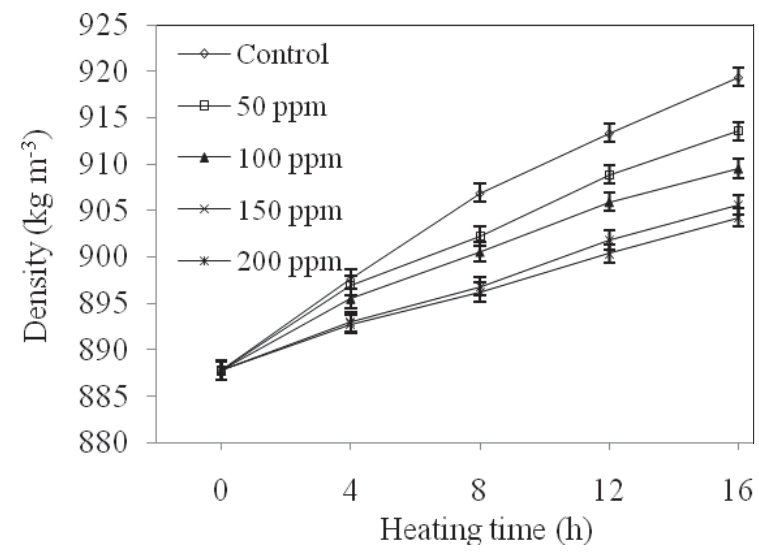

Fig. 1 - Variation of density as a function of heating time for control palm oil and its composition with different tertiary butyl hydroquinone content 


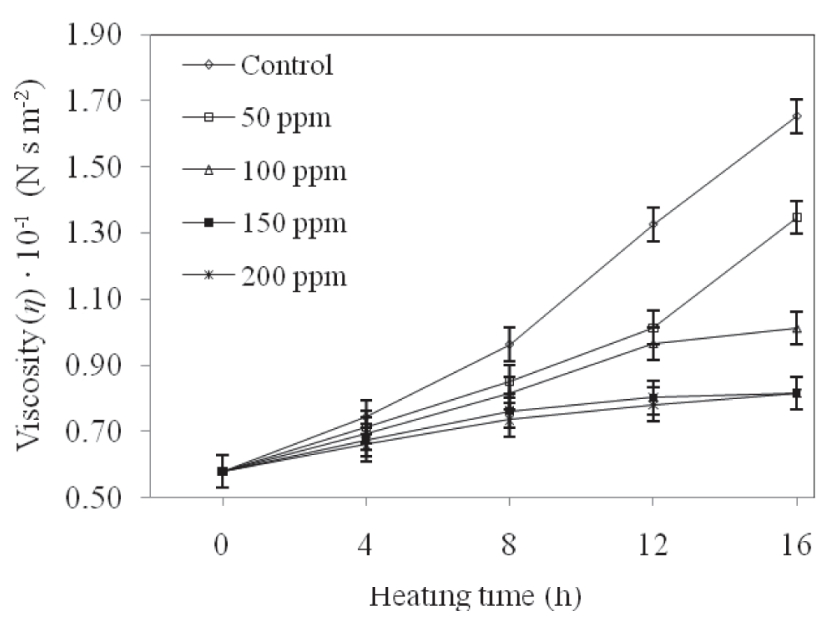

Fig. 2 -Viscosity as a function of heating time for the control and palm oil with different concentrations of tertiary butyl hydroquinone

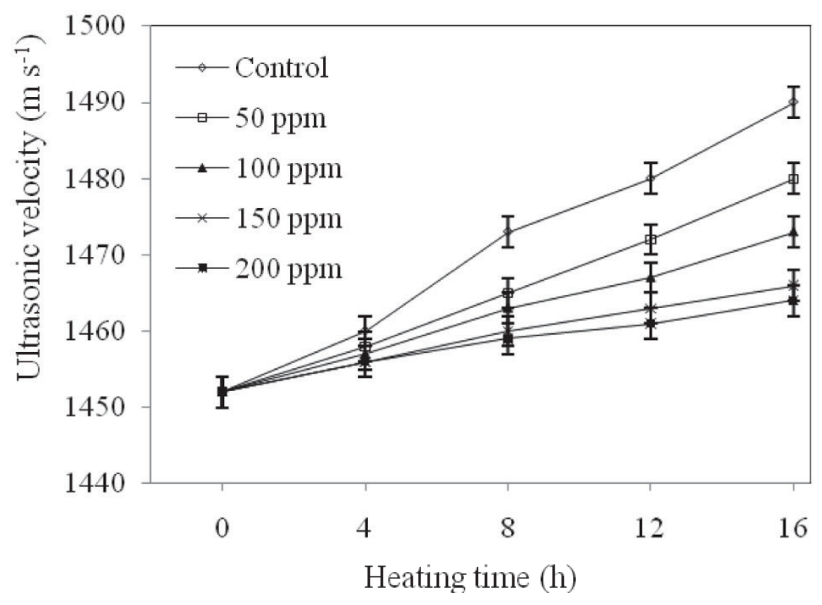

Fig. 3 - Effect of heating time on ultrasonic velocity of the control and palm oil with different concentrations of tertiary butyl hydroquinone

of $200 \mathrm{ppm}$ of TBHQ into the oil caused a slight increase in the density values as compared to the unheated oil. This may be due to slight changes in the chemical composition of oil formulations. The density of PO was $887.76 \mathrm{~kg} \mathrm{~m}^{-3}$ and changed to $919.38 \mathrm{~kg} \mathrm{~m}^{-3}$ and $904.23 \mathrm{~kg} \mathrm{~m}^{-3}$ for the heat-treated control oil and PO with 200 ppm TBHQ, respectively. The significant change in density of the control oil may be due to severe damage of its chemical structure and composition.

The changes in the viscosity values as a function of heating time are plotted in Fig. 2. The viscosity $(\eta)$ gradually and substantially increased with heating time. Adding 50, 100, 150 and $200 \mathrm{ppm}$ of TBHQ into PO led to different increments in the viscosity values for different oil heating times. The viscosity of fresh oil is $0.5785 \cdot 10^{-1} \mathrm{~N} \mathrm{~s} \mathrm{~m}^{-2}$. Fig. 2 shows that the heat-treated control oil exhibits a drastic increase in viscosity of $1.6523 \cdot 10^{-1} \mathrm{~N} \mathrm{~s} \mathrm{~m}^{-2}$, whereas the oil with $200 \mathrm{ppm}$ of TBHQ had the lowest viscosity of $0.84419 \cdot 10^{-1} \mathrm{~N} \mathrm{~s} \mathrm{~m}^{-2}$ for $16 \mathrm{~h}$ heating time. The obtained results were in good agreement with the already publishresults ${ }^{28,38-40}$. The viscosity of oil increases with duration of frying due to oxidation, isomerization, and polymerization reactions. An oxidation reaction leads to the formation of carbonyl or hydroxyl groups resulting in flux among the molecules that in turn increases the viscosity $^{33}$.

The ultrasonic velocity and attenuation depends on the physico-chemical properties of the oil ${ }^{41}$. A majority of the studies have used ultrasonic evaluation of food, as it is more reliable than attenuation and related to the physical and chemical properties of the medium ${ }^{42}$. The variation in the ultrasonic velocity with heating time is indicated in Fig. 3. After each heating period the ultrasonic velocity increases, and thus it is possible to distinguish the two oils $^{29}$. The changes in velocity of heat-treated oils are due to changes in the chemical composition of the oils. It was observed that the ultrasonic velocity increased linearly with heating time similar to the density and viscosity variations. The ultrasonic velocity increased significantly in the control oil from $1452 \mathrm{~m} \mathrm{~s}^{-1}$ to $1490 \mathrm{~m} \mathrm{~s}^{-1}$ after $16 \mathrm{~h}$ heat treatment. The smallest increase $\left(1464 \mathrm{~m} \mathrm{~s}^{-1}\right)$ in ultrasonic velocity was recorded for palm oil with $200 \mathrm{ppm}$ TBHQ.

Figs. 4 to 7 illustrate the interaction between the ultrasonic waves and the composition of oil molecules. The adiabatic compressibility and free length are the deciding factors of the ultrasonic velocity in liquid systems ${ }^{29}$. The increase in the ultrasonic velocity was due to the decrease in the free length and adiabatic compressibility. The adiabatic compressibility and free length were found to differ slightly for oil with $200 \mathrm{ppm}$ of TBHQ as compared to the changes in the base oil. The decrease in the values of the free length indicates that the triglyceride molecule with unsaturated bonds is likely to collapse as a result of the oxidation process ${ }^{32}$. The structures of triglycerides are kept intact due to the presence of $\mathrm{TBHQ}^{41}$ in oil. Hence, the values of free length, which indicates the intermolecular distance, did no reduce as a result of using TBHQ.

The changes in the relaxation time of oil against heating time are plotted in Fig. 6. It was found that relaxation time increased with the heating time. The base oil showed greater enhancement in relaxation time, and its lowest increment was found in oil with $200 \mathrm{ppm}$ of TBHQ. The dispersion of ultrasonic velocity in the system should contain information about the characteristic time, $\tau$ of the relaxation process that causes dispersion, where, $\tau$ is in the order of $1 \cdot 10^{-11} \mathrm{~s}$ due to the structural relaxation process $^{44}$ and in such a situation, the molecules rearrange due to a co-operative process ${ }^{45}$. 


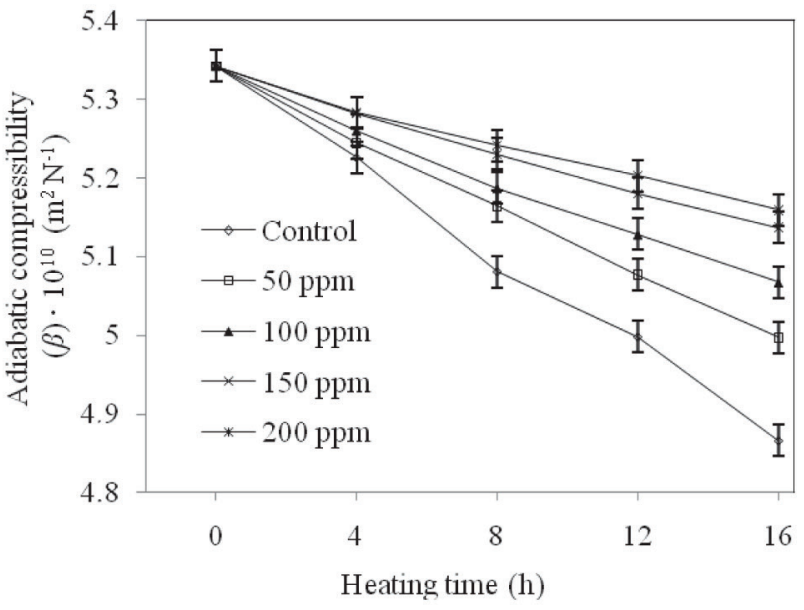

Fig. 4 -Variation of adiabatic compressibility of the control and palm oil with different concentrations of tertiary butyl hydroquinone with heating time

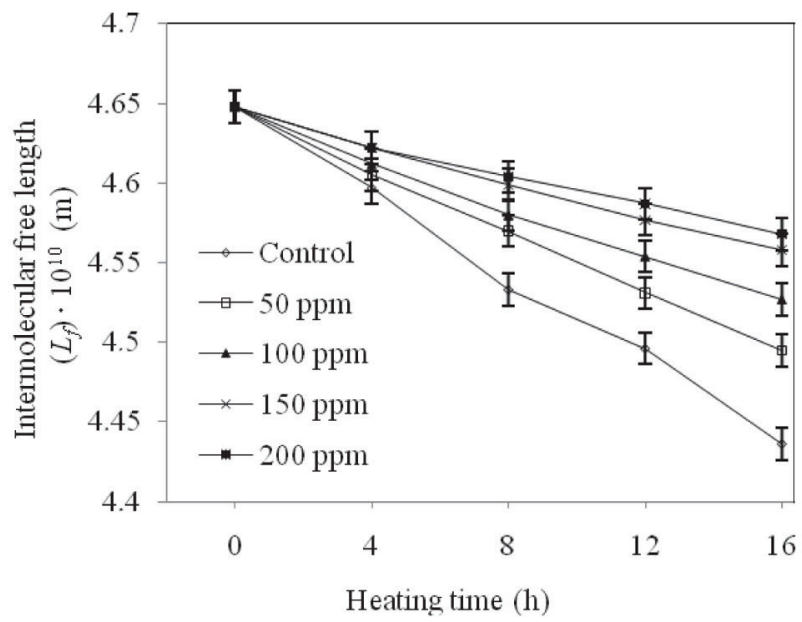

Fig. 5 - Effect of heating time on intermolecular free length of the control and palm oil with different concentrations of tertiary butyl hydroquinone

The acoustic impedance is calculated using ultrasonic velocity and density, and was plotted as a function of heating time (Fig. 7). It was found that the acoustic impedance was low in the oil with TBHQ and without heating. With increased heating time, the acoustic impedance increased in the base oil, but to a very little extent in the oil with 200 ppm of TBHQ. The excess parameters play a major role in understanding the nature of intermolecular interactions in liquid mixtures ${ }^{46}$. The intermolecular free length and acoustical impedance depend upon the intermolecular attractive and repulsive forces. Excess acoustical impedance may be due to the pyrolysis effect allowing the fitting of molecules of different sizes after collapsing the triglyceride structure.

The measured acoustic parameters, such as $\beta, \tau$, $L_{f}$ and $Z$, were correspondingly found to change to a large extent in the control PO. The addition of the

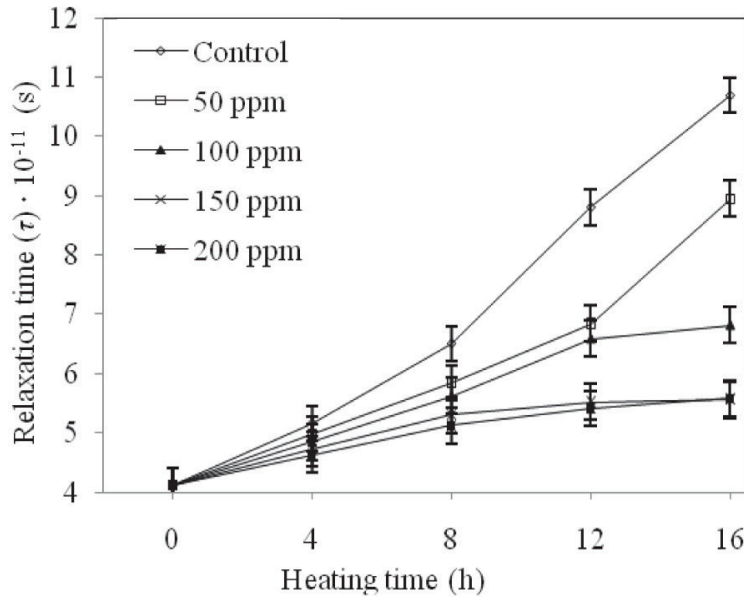

Fig. 6 -Variation of relaxation time of the control and palm oil with different concentrations of tertiary butyl hydroquinone as a function of heating time

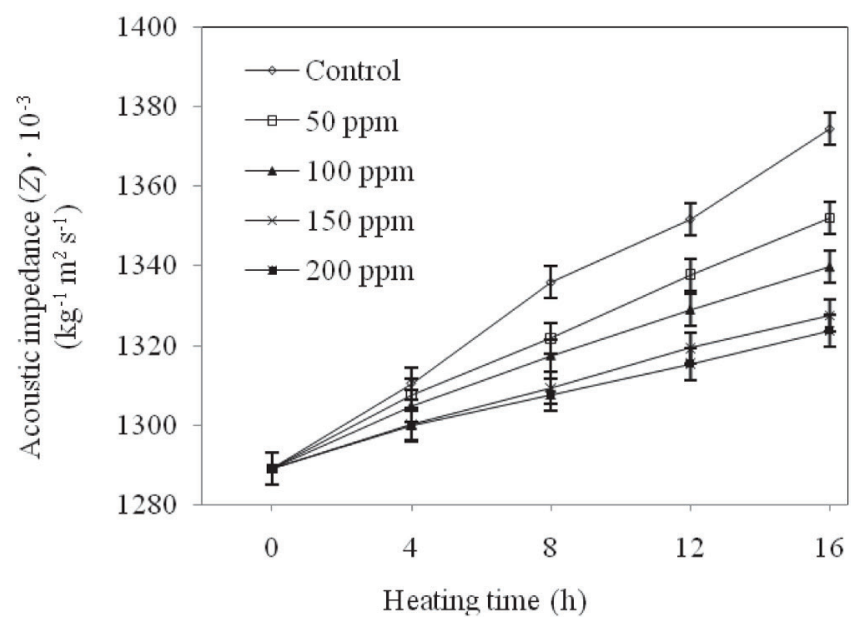

Fig. 7 - Variation of acoustic impedance of the control and palm oil with different concentrations of tertiary butyl hydroquinone as a function of heating time

antioxidant, TBHQ, allowed no breaking up of the molecular clustering in the oil. The interaction between the molecules of the oil occurred to a lesser extent, and hence, very small structural changes occurred during heating in the presence of an antioxidant. In the control oil, as the antioxidant activity is much less, a break up occurred in the molecular clustering, releasing several dipoles for the interaction. In view of the greater interactions, larger changes have occurred for heat-treated oils in the adiabatic compressibility, intermolecular free length, relaxation time, and acoustical impedance. It was found that the stability of PO with antioxidant TBHQ was better even at high temperatures and after prolonged heating ${ }^{31,32}$.

Gas chromatography (GC) is a useful analytical technique for studying the effect of different processing conditions on the fatty acid composition of oils ${ }^{47}$. Tables 1 and 2 present the results of the fatty 
Table 1 -Changes in the fatty acid composition (\%) of palm oil during heating

\begin{tabular}{|c|c|c|c|c|c|}
\hline \multirow{2}{*}{ Fatty acid } & \multicolumn{5}{|c|}{ Heating time (h) } \\
\hline & 0 & 4 & 8 & 12 & 16 \\
\hline $\mathrm{C} 14: 0$ & $1.7 \pm 0.2$ & $2.3 \pm 0.3$ & $3.6 \pm 0.4$ & $4.1 \pm 0.3$ & $4.5 \pm 0.2$ \\
\hline C16:0 & $44.3 \pm 1.2$ & $45.0 \pm 1.1$ & $47.1 \pm 1.0$ & $48.9 \pm 0.9$ & $50.8 \pm 1.2$ \\
\hline C18:0 & $4.8 \pm 0.4$ & $5.0 \pm 0.5$ & $6.7 \pm 0.4$ & $8.3 \pm 0.5$ & $10.9 \pm 0.6$ \\
\hline C18:1 & $38.4 \pm 1.1$ & $38.8 \pm 0.9$ & $34.4 \pm 0.8$ & $31.2 \pm 0.7$ & $27.3 \pm 0.8$ \\
\hline $\mathrm{C} 18: 2$ & $10.8 \pm 0.8$ & $8.9 \pm 0.7$ & $8.2 \pm 0.6$ & $7.5 \pm 0.3$ & $6.5 \pm 0.3$ \\
\hline $\mathrm{C} 18: 3$ & ND & ND & ND & ND & ND \\
\hline Total & 100 & 100 & 100 & 100 & 100 \\
\hline SFA, $\%$ & $50.8 \pm 1.8$ & $52.3 \pm 1.2$ & $57.4 \pm 1.2$ & $61.3 \pm 1.1$ & $66.2 \pm 1.1$ \\
\hline MUFA, \% & $38.4 \pm 1.1$ & $38.8 \pm 0.9$ & $34.4 \pm 0.8$ & $31.2 \pm 0.7$ & $27.3 \pm 0.8$ \\
\hline PUFA, \% & $10.8 \pm 0.8$ & $8.9 \pm 0.7$ & $8.2 \pm 0.6$ & $7.5 \pm 0.3$ & $6.5 \pm 0.3$ \\
\hline
\end{tabular}

C14:0 Myristic acid, C16:0, palmitic acid; C18:0, stearic acid; C18:1, oleic acid; C18:2, linoleic acid; $\alpha$-C18:3, $\alpha$-linolenic acid, SFA saturated fatty acids, MUFA monounsaturated fatty acids, PUFA polyunsaturated fatty acids, ND not detected

Table 2 - Changes in the fatty acid composition (\%) of palm oil with 200 ppm TBHQ during heating

\begin{tabular}{l|c|c|c|r|r}
\hline \multirow{2}{*}{ Fatty acid } & \multicolumn{5}{c}{ Heating time $(\mathrm{h})$} \\
\cline { 2 - 6 } C14:0 & 0 & 4 & 8 & 12 & 16 \\
C16:0 & $1.7 \pm 0.2$ & $1.9 \pm 0.2$ & $2.1 \pm 0.4$ & $2.3 \pm 0.2$ & $2.5 \pm 0.2$ \\
C18:0 & $44.3 \pm 1.2$ & $44.6 \pm 1.0$ & $45.0 \pm 1.0$ & $45.4 \pm 0.9$ & $45.8 \pm 1.0$ \\
C18:1 & $4.8 \pm 0.4$ & $5.1 \pm 0.4$ & $7.4 \pm 0.4$ & $9.7 \pm 0.5$ & $11.4 \pm 0.5$ \\
C18:2 & $38.4 \pm 1.1$ & $38.6 \pm 0.8$ & $36.6 \pm 0.9$ & $34.3 \pm 0.8$ & $32.8 \pm 1.2$ \\
C18:3 & $10.8 \pm 0.8$ & $9.8 \pm 0.7$ & $8.9 \pm 0.5$ & $8.3 \pm 0.3$ & $7.5 \pm 0.4$ \\
Total & $\mathrm{ND}$ & $\mathrm{ND}$ & $\mathrm{ND}$ & $\mathrm{ND}$ & $\mathrm{ND}$ \\
SFA, \% & 100 & 100 & 100 & 100 & 100 \\
MUFA, \% & $50.8 \pm 1.8$ & $51.6 \pm 1.2$ & $54.5 \pm 1.2$ & $57.4 \pm 1.2$ & $61.7 \pm 1.2$ \\
PUFA, \% & $38.4 \pm 1.1$ & $38.6 \pm 0.8$ & $36.6 \pm 0.9$ & $34.3 \pm 0.8$ & $32.8 \pm 1.2$ \\
\hline C14:0 Mytic & $10.8 \pm 0.8$ & $9.8 \pm 0.7$ & $8.9 \pm 0.5$ & $8.3 \pm 0.3$ & $7.5 \pm 0.4$ \\
\hline
\end{tabular}

C14:0 Myristic acid, C16:0, palmitic acid; C18:0, stearic acid; C18:1, oleic acid; C18:2, linoleic acid; $\alpha$-C18:3, $\alpha$-linolenic acid, SFA saturated fatty acids, MUFA monounsaturated fatty acids, PUFA polyunsaturated fatty acids, ND not detected

acid (FA) profiles of PO and formulated PO, respectively with heating time. The incorporation of TBHQ enhanced the oxidative stability of the heated oil samples. The estimated concentration of the unsaturated fatty acids by means of relative GC peak areas showed that palm oil treated with 200 ppm TBHQ had significantly lower values in comparison with the base oil. The PO with 200 ppm TBHQ showed slight changes in the FA composition in heated oils, which reflected that antioxidant addition in unsaturated oils retards the degradation of oils. A reduction in the amount of linoleic acid was observed with the increase in concentration of oleic acid ${ }^{18}$

The FA composition of oil has significant effect on its frying performance as well as on its physi- co-chemical behaviours. The FA profile of the frying oils changed as a result of cyclization, polymerization, and hydrolytic, oxidative and other chemical reactions promoted by frying conditions ${ }^{48}$. The linoleic acid level in deep-frying oils appears to be an obviously negative factor in oil stability. In this study, changes in the FA profile of oils during frying are basically among the unsaturated fatty acids, whereas the saturated fatty acids are slightly increased.

Pure palm oil contained myristic acid, palmitic acid; stearic acid; oleic acid; linoleic acid in the proportions of $1.7,44.3,4.8,38.4$ and $10.8 \%$, respectively. The compositions of myristic acid, palmitic acid, stearic acid, oleic acid, and linoleic acid, in heat-treated base oils changed to 4.5, 50.8, 10.9, 
Table 3 -Variation of $\%$ free fatty acid and \% total polar compounds in

\begin{tabular}{c|cc|c|c}
\hline \multicolumn{3}{c}{ PO and PO + 200 ppm TBHQ for different heating times } \\
\hline \multirow{2}{*}{$\begin{array}{c}\text { Heating } \\
\text { time (h) }\end{array}$} & \multicolumn{2}{|c|}{$\%$ FFA } & \multicolumn{2}{c}{$\%$ TPC } \\
\cline { 2 - 5 } & PO & PO + TBHQ & PO & PO + TBHQ \\
\hline 0 & 0.16 & 0.16 & 5.0 & 5.0 \\
4 & 0.25 & 0.20 & 8.1 & 7.2 \\
8 & 0.33 & 0.24 & 12.1 & 8.9 \\
12 & 0.41 & 0.28 & 18.1 & 10.1 \\
16 & 0.51 & 0.32 & 22.3 & 11.5 \\
\hline
\end{tabular}

$27.3 \%$ and $6.5 \%$, respectively, and in oil with 200 ppm TBHQ, it changed to $2.5 \%, 45.8 \%, 11.4 \%$, $32.8 \%$ and $7.5 \%$, respectively after $16 \mathrm{~h}$ of heating. The frying stability of PO can be improved by incorporation of TBHQ, in order to reduce the change in linoleic and oleic acid, and consequently to improve their oxidative and heat stabilities. The data indicates that the amount of unsaturated fatty acid decreased gradually during repeated deep fat frying cycles. This may be due to oxidative and thermal degradation reactions during repeated deep fat frying cycles of the unsaturated fatty acid constituents of triacylglycerols ${ }^{44}$. Better oxidation stabilities were found in the formulated oils during repeated frying as compared to the base oil.

Formation of free fatty acids (FFA) for heat-treated oil is considered to be a measure of rancidity of oils. The percentage of FFA formed for repeatedly heat-treated oil is presented in Table 3. Initially, the base oil and the TBHQ-loaded oils had a similar FFA content; as the heating time continued the palm oil showed a higher FFA value than the TBHQ-loaded oils. FFA is formed due to hydrolysis of triglycerides and may be promoted by the reaction of oil with moisture ${ }^{49}$. Addition of antioxidant caused a significant reduction in FFA values of PO. FFA content is the frequently used data to probe the shelf life of frying oil, but it is not recommended to be the only indicator.

Generally, degradation of oil during frying is accompanied increased polar compounds in the oils $^{50}$. Many researchers have reported that total polar compounds (TPC) are the most reliable indicator of oil degradation ${ }^{29,51}$. Polar compounds include all oxidized triglycerides, dimerized triglycerides, FFAs, monoglycerides, diglycerides, sterols, antioxidants, antifoamers, hydrogenation catalyst residues and soaps ${ }^{7}$. Table 3 shows the percentage of TPC formed during heating in $\mathrm{PO}$ and $\mathrm{PO}$ with $200 \mathrm{ppm}$ TBHQ. Initially, the TPC contents of the base oil and the formulated oil were similar. The increase in the rate of TPC formation with heating time in the base oil was different from the formulated oil with TBHQ. After $16 \mathrm{~h}$ of heating, the final TPC values were $22.3 \%$ in the base oil and $11.5 \%$ in the PO+ $200 \mathrm{ppm}$ TBHQ. These parameters are indicators of the state of oil deterioration. These results clearly indicate that the PO with $200 \mathrm{ppm}$ TBHQ was more stable chemically than PO. Polar compounds are the sum of non-triglycerides of oil including fatty acids, sterols, tocopherols, mono- and di-triglycerides, alcohols, aldehydes, ketones, and other soluble compounds in oil that are more polar than triglycerides ${ }^{52}$. Polar compounds accumulate on the surface of the frying pan and foods during frying. Most of the poisonous materials are considered to exist in the polar compounds of oil ${ }^{53}$ formed during oxidation. If the TPC exceeds $25 \%$, according to FDA norms, the oil should be disposed ${ }^{54}$. It is observed that after 16 $\mathrm{h}$ of heating, polar compounds in the PO reached $22.3 \%$, and this may exceed $25 \%$ on further heating. On the other hand, PO with $200 \mathrm{ppm}$ of TBHQ reached $11.5 \%$, may not have polar compounds exceeding $25 \%$ on further heating, and hence can be used.

\section{Correlation of ultrasonic velocity with total polar compounds and free fatty acids}

As long as the ultrasonic velocity is related to the physical properties of the medium like viscosity and density, velocity should also be related to these chemical indicators. The relationship between the ultrasonic velocity and both TPC and FFA for heated PO and PO with 200 ppm TBHQ at $30{ }^{\circ} \mathrm{C}$ are shown in Figs. 8 and 9, respectively. The changes in polar compounds with the change in ultrasonic velocity relationships are found to be linear with the regression value, $R^{2}=0.982$ in the case of base oil, and $R^{2}=0.996$ for TBHQ-treated oil ${ }^{29}$. The changes in FFA with change in ultrasonic velocity relationships are also found to be more linear with regression value, $R^{2}=0.991$ in the case of base oil, and $R^{2}$ $=0.983$ for TBHQ-treated oil. These findings are in accordance with data found elsewhere ${ }^{29,42}$. These chemical parameters, widely considered as oil quality indicators and polynomial fits were found when relating the velocity. Therefore, measurement of ultrasonic velocity permits an estimation of the percentages of polar compounds and FFA independently in the oil.

\section{FT-IR studies}

The characteristics of FTIR for fresh PO, heated PO with and without antioxidant are shown in Fig. 10. The band at $3010 \mathrm{~cm}^{-1}$ is attributed to $\mathrm{C}-\mathrm{H}$ stretching vibration of the cis-double bond ${ }^{55}$. Strong band absorptions were observed in the region $3000-2800 \mathrm{~cm}^{-1}$ corresponding to $\mathrm{C}-\mathrm{H}$ stretching 
vibrations. The stretching vibrations of methylene $\left(-\mathrm{CH}_{2}-\right)$ and methyl $\left(-\mathrm{CH}_{3}\right)$ groups can be seen at 2923 and $2854 \mathrm{~cm}^{-1}$, respectively. The spectral

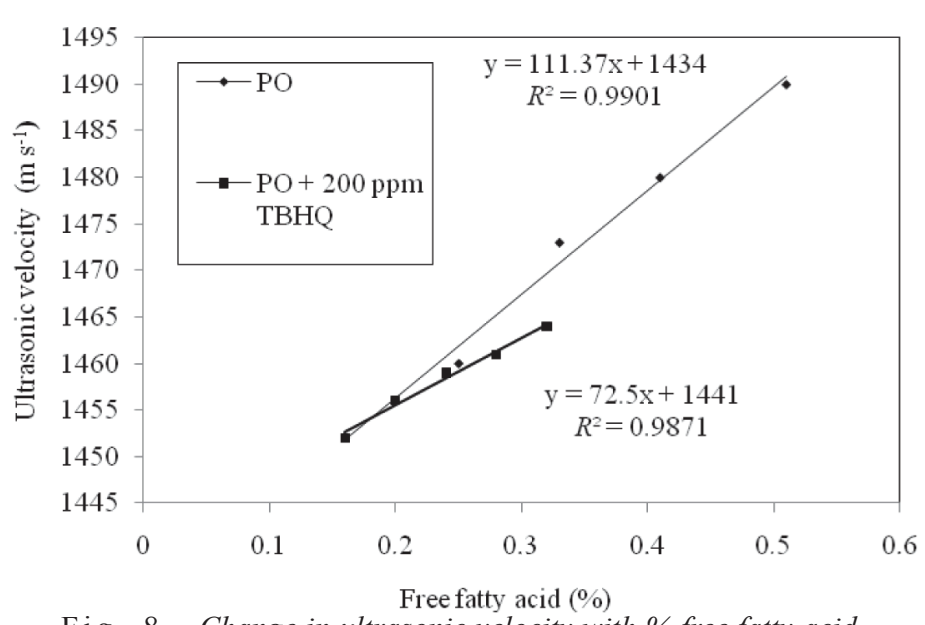

Fig. 8 - Change in ultrasonic velocity with \% free fatty acid

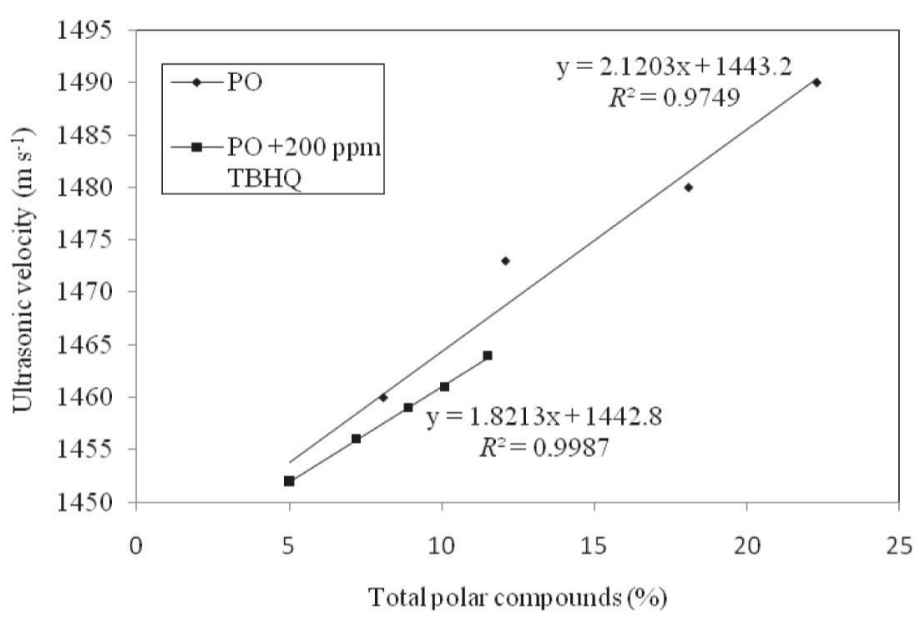

Fig. 9 - Change in ultrasonic velocity with \% total polar compounds

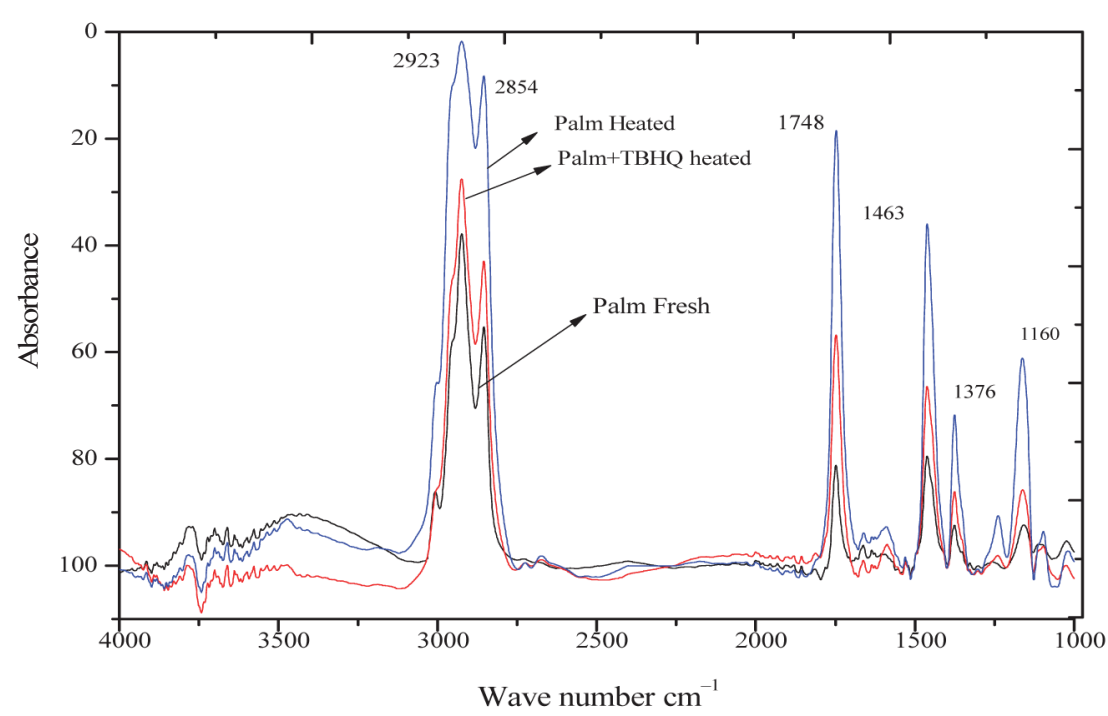

Fig. 10 - FTIR spectra of fresh palm oil, heated palm oil, and heated palm oil with $T B H Q$ region between 3050 and $2740 \mathrm{~cm}^{-1}$ undergoes several changes during the thermal oxidation process.

The changes in the FTIR spectra were observed for 16 hat $180{ }^{\circ} \mathrm{C}$ heated PO. The band at $2854 \mathrm{~cm}^{-1}$ and the shoulder at $2960 \mathrm{~cm}^{-1}$ increases their intensity and width for $16 \mathrm{~h}$ heated PO at $180^{\circ} \mathrm{C}$. Significant changes are noticed in heated base oil as compared to the heated PO with TBHQ. Methylene and methyl groups are also observed at $1463 \mathrm{~cm}^{-1}$ and $1376 \mathrm{~cm}^{-1}$ due to their bending vibrations.

Two absorption peaks observed at 1748 and $1160 \mathrm{~cm}^{-1}$ are due to the stretching vibrations of the aldehyde group $(\mathrm{C}=\mathrm{O})$ and ester group $(\mathrm{C}-\mathrm{O})$ respectively ${ }^{56}$. In the region of the former peak, infrared energy is absorbed due to the carbon-oxygen bonds in the oil, and it is often used for determining the level of oxidation.

The peak area around $1748 \mathrm{~cm}^{-1}$ is due to $\mathrm{C}=\mathrm{O}$ bond stretching vibration. It is also interesting to follow the spectral changes in the $\mathrm{C}=\mathrm{O}$ region $\left(\sim 1748 \mathrm{~cm}^{-1}\right)$. Here, the study shows changes in peak are at $1748 \mathrm{~cm}^{-1}$ for the heated samples. This observation is due to production of saturated aldehyde functional groups or other secondary oxidation products that cause an absorbance at $1728 \mathrm{~cm}^{-1}$, which overlaps with the stretching vibration at 1746 $\mathrm{cm}^{-1}$ of the ester carbonyl functional group of the triglycerides. When new carbonyls are formed from initial aldehyde and ketone compounds, the maximum absorbance is in the region between 1720 and $1760 \mathrm{~cm}^{-1}$ resulting in a broadening of the $1748 \mathrm{~cm}^{-1}$ band. The total amount of carbonyls formed can be measured by the intensity and area of the $1748 \mathrm{~cm}^{-1}$ band. Also, it observed that height of the peaks in the heated PO is four times that of fresh PO at the regions 1463,1376 and $1160 \mathrm{~cm}^{-1}$ and it is two times in the case of heated PO with TBHQ. The increases in the intensity of peaks indicate the changes in the chemical composition, and in turn, it is a sign of deterioration ${ }^{47}$.

\section{Conclusion}

In the current study, a method has been developed to make use of ultrasonic velocity to determine the thermal stability of palm oil. Incorporation of varying amounts of TBHQ $(50,100$, 150 and $200 \mathrm{ppm}$ ) into PO provides improvements in the antioxidative potency. The addition of antioxidant, TBHQ at the level of $200 \mathrm{ppm}$, resulted in the retardation of oxidative deterioration of PO. Also in the present 

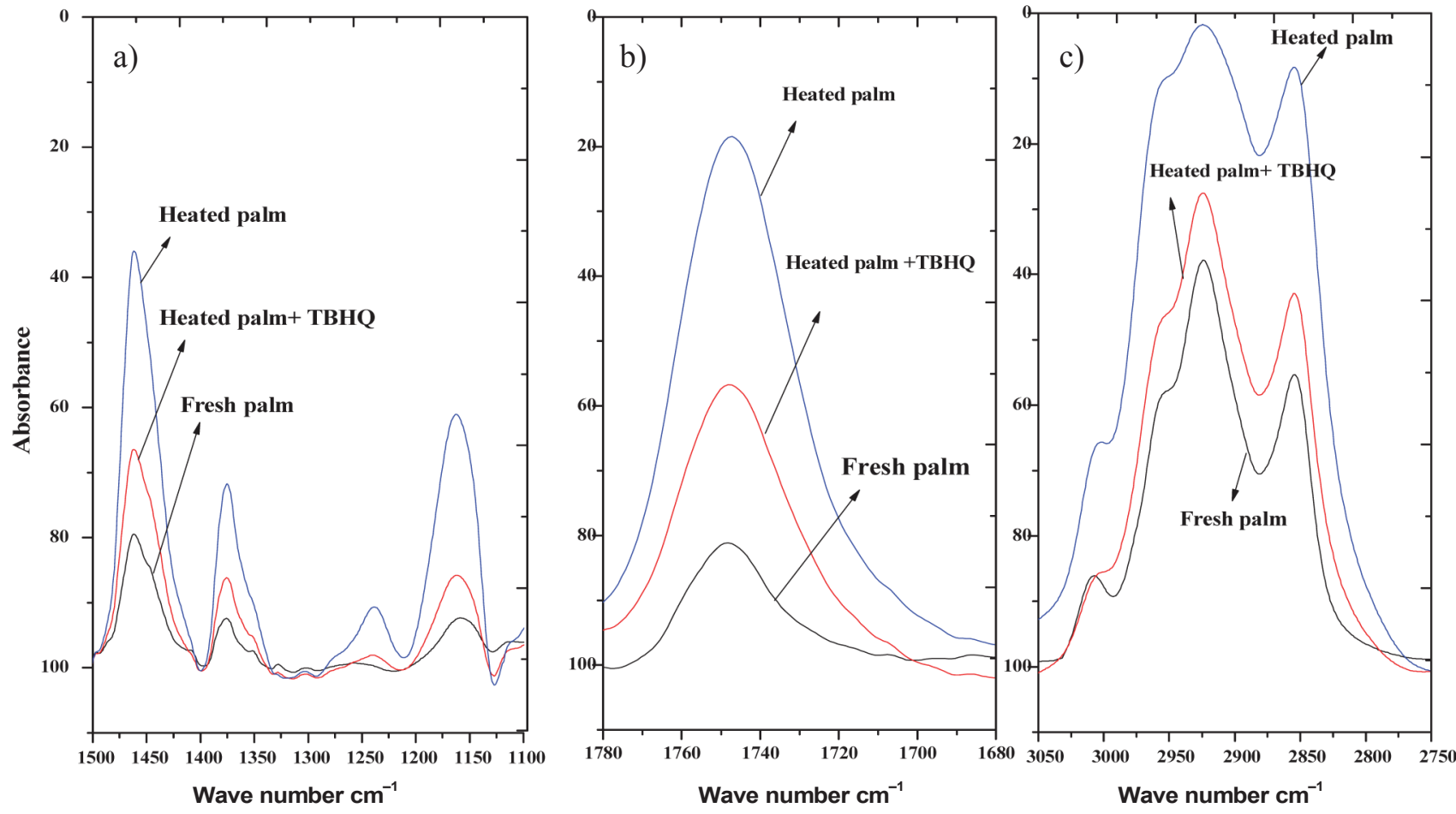

Fig. 11 - FTIR spectra of fresh palm oil and heated palm oil with and without TBHQ; (a) $1500 \mathrm{~cm}^{-1}$ to $1100 \mathrm{~cm}^{-1}$, (b) 1780 to $1680 \mathrm{~cm}^{-1}$ and (c) 3050 to $2750 \mathrm{~cm}^{-1}$

study, the variation in the secondary parameters $\beta, \tau$, $L_{f}$ and $Z$ of palm oil with and without TBHQ are compared. It is found that the unsaturated fatty acid composition of the oil did not become saturated due to the presence of the antioxidant (TBHQ) upon repeated heating and at $200 \mathrm{ppm}$ of TBHQ loaded formulation stability is effectively retained, which is also in line with the general standard for food additives. These secondary parameters clearly indicate that the state of deterioration is worse in PO compared to PO with TBHQ. Also, it was found that there is a linear change in the FFA and TPC along with change in ultrasonic velocity as a function of heating time. Fatty acids profile obtained by GC and changes in FTIR spectral regions 3050-2800 $\mathrm{cm}^{-1}$ and $1100-1745 \mathrm{~cm}^{-1}$ supports the data obtained by ultrasonic studies. Palm oil with TBHQ has better thermal stability than PO alone. Ultrasonic velocity and acoustic parameters can be used to assess the thermal stability of PO. This methodology could be useful in evaluating the oxidative stability of edible oils in a simple and fast manner.

\section{References}

1. Lambelet, P., Grandgirard, A., Gregoire, S., Formation of modified fatty acids and oxyphytosterols during refining of low erucid acid rapeseed oil, Journal of Agricultural and Food Chemistry 52 (2003) 4284.

2. Ježek, D., Brnčić, M., Rima, B. S., Karlović S., Bosiljkov, T., Tripalo B., Karlović, D., Šipušić, J., Pukec, D., Porosity of deep fried breaded chicken meat, Food Technology and Biotechnology 47(4) (2009) 38 .
3. Sunga, H.-J., Songa, W.-J., Kimb, K.-P., Ryua, S., Kang, D.-H., Combination effect of ozone and heat treatments for the inactivation of Escherichia coli O157:H7, Salmonella typhimurium, and Listeria monocytogenes in Apple juice, International Journal of Food Microbiology 171 (2014) 147. doi: https://doi.org/10.1016/j.ijfoodmicro.2013.11.001

4. Kaya, Z., Yıldiz, S., Ünlütürk, S., Effect of UV-C irradiation and heat treatment on the shelf life stability of a lemonmelon juice blend: multivariate statistical approach, Innovative Food Science and Emerging Technologies 29 (2015) 230.

doi: https://doi.org/10.1016/j.ifset.2015.03.005

5. Rossell, J. B., Frying improving quality. Part II, Woodhead Publishing Limited. Cambridge, UK, (2001).

6. Nor Hayati, I., Aminah A., Mamot, S., Physical characteristics of modified milk fat in high melting fat preparation, International Journal of Food Sciences and Nutrition $\mathbf{5 3}$ (2002) 43. doi: https://doi.org/10.1080/09637480120057000

7. Litwinienko, G., Kasprzsycka-Guttman, T., Jamanek, D., DSC study of antioxidant properties of dihydroxyphenols, Termochimica Acta 331 (1999) 79. doi: https://doi.org/10.1016/S0040-6031(99)00058-1

8. de Guzman, R., Tang, H., Salley, S., Ng, K. Y. S., Synergistic effects of antioxidants on the oxidative stability of soy bean oil- and poultry fat-based biodiesel, Journal of American Oil Chemical Society 86 (2009) 459. doi: https://doi.org/10.1007/s11746-009-1373-8

9. Pimpa, Duangkhae Kanjansopa, Sophan Boonlam, Effect of addition of antioxidants on oxidative stability of refined bleached and deodorized palm oil, Kasetstart Journal 43 (2009) 370.

10. Zhang, Y., Yang, L., Zu, Y., Chen, X., Wang, F., Liu, F., Oxidative stability of sunflower oil supplemented with carnosic acid compared with synthetic antioxidants during accelerated storage, Food Chemistry 118 (2010) 656. doi: https://doi.org/10.1016/j.foodchem.2009.05.038 
11. Assimopoulou, A. N., Zlatanos, S. N., Papageorgiou, V. P., Antioxidant activity of natural resins and bioactive triterpenes in oil substrates, Food Chemistry 92 (2005) 721. doi: https://doi.org/10.1016/j.foodchem.2004.08.033

12. Erkan, N., Ayranci, G., Ayranci, E., A kinetic study of oxidation development in sunflower oil under microwave heating: effect of natural antioxidants, Food Research International 42 (2009) 1171. doi: https://doi.org/10.1016/j.foodres.2009.06.003

13. Goli, A. H., Barzegar, M., Sahari, M. A., Antioxidant activity and total phenolic compounds of pistachio (Pistachia vera) hull extracts, Food Chemistry 92 (2005) 521. doi: https://doi.org/10.1016/j.foodchem.2004.08.020

14. Thaipong, K., Boonprakob, U., Crosby, K., Cisneros-Zevallos L., Byrne, D. H., Comparison of ABTS, DPPH, FRAP and ORAC assays for estimating antioxidant activity from guava fruit extracts, Journal of Food Composition and Analysis 19 (2006) 669. doi: https://doi.org/10.1016/j.jfca.2006.01.003

15. Ahmadi, F., Kadivar, M., Shahedi, M., Antioxidant activity of Kelussia odoratissima Mozaff in model and food systems, Food Chemistry 105 (2007) 57. doi: https://doi.org/10.1016/j.foodchem.2007.03.056

16. Valenzuela, A., Sanhueza, J., Nieto, S., Cholesterol oxidation: Health hazard and the role of antioxidants in prevention, Biological Research 36(3-4) (2003) 291.

17. Kishk, Y. F. M., Al-Sayed, H. M., Free-radical scavenging and antioxidative activities of some polysaccharides in emulsions, LWT - Food Science and Technology 40(2) (2007) 270.

18. Akhtar, H., Tariq, I., Mahmood, S., Hamid, S., Khanum, R., Effect of antioxidants on stability, nutritional values of refined sunflower oil during accelerated storage and thermal oxidation in frying, Bangladesh Journal of Scientific and Industrial Research 47(2) (2012) 223.

19. Maisuthisakul, P., Gordon, M. H., Pongsawatmanit, R., Suttajit, Enhancing the oxidative stability of rice crackers by addition of the ethanolic extract of phytochemicals from Cratoxylum formosum Dyer, M. Asia Pacific Journal of Clinical Nutrition, 16 (Suppl 1) (2007) 37.

20. Mishra, B. R., Sharma, H. K., Sengar, G., Quantification of rice bran oil in the blended oils, Grassas y Acietes 63(1) (2012) 53 doi: https://doi.org/10.3989/gya.033311

21. Gulla, S., Waghray, K., Effect of storage on physico-chemical characteristics and fatty acid composition of selected oil blends, Journal of Life Science 3(1) (2011)35.

22. Barison, A., da Silva, C. W. P., Campos, F. R., A simple methodology for the determination of fatty acid composition in edible oils through $1 \mathrm{H}$ NMR spectroscopy, Magnetic Resonance in Chemistry 48(8) (2010) 642.

23. Knothe, G., Kenar, J. A., Determination of the fatty acid profile by $1 \mathrm{H}-\mathrm{NMR}$ spectroscopy, European Journal of Lipid Science and Technology 106(2) (2004) 88. doi: https://doi.org/10.1002/ejlt.200300880

24. Rao, H., Fullana, A., Sidhu, S., Carbonell-barrachina, Á. Emissions of volatile aldehydes from heated cooking oils. Food Chemistry 120(1) (2010) 59. doi: https://doi.org/10.1016/j.foodchem.2009.09.070

25. Karlović, S., Bosiljkov T., Brnčić M., Semenski, D., Dujmić, $F$., Tripalo B., Ježek D., Reducing fat globules particle-size in goat milk: ultrasound and high hydrostatic pressures approach, Chemical and Biochemical Engineering Quarterly 28 (4) (2014) 499.

doi: https://doi.org/10.15255/CABEQ.2014.19400
26. Brnčić, M., Karlović, S., Rimac Brnčić, S., Penava, A., Bosiljkov, T., Ježek, D., Tripalo, B., Textural properties of infra-red dried apple slices as affected by high power ultrasound pre-treatment, African Journal of Biotechnology 9 (41) (2010) 6907

27. Zawieja, Wolny, L., Ultrasonic disintegration of sewage sludge to increase biogas generation, Chemical and Biochemical Engineering 27(4) (2013) 491.

28. Niñoles, L., Mulet, A., Ventanas, S., Benedito, Ultrasonic assessment of the melting behaviour in fat from Iberian dry-cured hams, Meat Science 85(1) (2010) 26. doi: https://doi.org/10.1016/j.meatsci.2009.11.018

29. Izbaim, B. D., Faiz, B., Moudden, A., Taifi, N., Aboudaovd, I., Evaluation of the performance of Frying Oils using an ultrasonic technique, International Journal Grasas y Aceites 61(2) (2010)151. doi: https://doi.org/10.3989/gya.087709

30. Sarmento, C. M. P., Ferriera, S. R. S., Henseh, Supercritical fluid extraction (SFE) of rice bran oil to obtain fractions enriched with tocopherols and tocotrienols, Brazilian Journal of Chemical Engineering 23 (2006) 243.

31. Rehab F. M. Ali, Improvement in the stability of fried sunflower oil by using different levels of pomposia (Syzyygium cumini), Electronic Journal of Environmental Agriculture and Food Chemistry 9(2) (2010) 396

32. Okoye, I. P., Onwe, O. J., Aakarnta, O. J., The effect of the antioxidant quercetin on the oxidation stability of lubricating oil, Scientia Africana 8(2) (2009) 26.

33. Valantina, S., Sahayararaj, P., Prema, A., Antioxidant stability in palm and rice bran oil using simple parameters. Journal RASAYAN Chemistry 3(2010)44.

34. Kesavasamy, R., Ponnuswamy, V., Kannappan, A. N., Molecular interaction studies in the ternary liquid mixture of pyridine + benzene $+\mathrm{n}$, n-dimethylformamide, ARPN Journal of Engineering and Applied Science 3(4) (2008) 41.

35. Priva, C., Nithya, S., Velraj A. N., Molecular interaction studies in liquid mixture using ultrasonic technique, Journal of Advanced Science and Technology 18 (2010) 59.

36. Ahmed Tarmizi, A., Razati Ismail, Comparison of the frying stability of standard palm olein and special quality palm olein, Journal of American Oil Chemical Society 85 (2008) 254.

37. Firestone, D., (Ed.) Method no: Ce1-62. Official methods and recommended practices of the American Oil of Chemical Society, $5^{\text {th }}$ edn, USA (1998).

38. Farag, R. S., El-Baroty, G. S., Amany, The influence of phenolic extracts obtained from the olive plants (cvs. Picual and Kronakii) on stability of sunflower oil, Journal of Food Science and Technology 38 (2003) 81. doi: https://doi.org/10.1046/j.1365-2621.2003.00665.x

39. Shaker, E. S., Antioxidative effect of extracts from grape seed and peel on lipid oxidation in oils of sunflower, LWT Journal of Food Science and Technology 39 (2006) 883.

40. Anany, A., Influence of pomegranate (Punica granatum) peels extract on the stability of sunflower oil during deepfat frying process, Electronic Journal of Food and Plants Chemistry 1 (2007) 14

41. McClements, D. J., Ultrasonic characterization of food and drinks: Principles, methods and applications Crit. Rev., Food Science 37 (1997) 1.

42. Benedito, J., Garcia-Perez, J. V., Dobarganes, M. C., Rapid evaluation of frying oil degradation using ultrasonic technology, Food Research International 40(3) (2007) 406. doi: https://doi.org/10.1016/j.foodres.2006.10.017

43. de Guzman R., Haiying Tang, Salley, S., Synergistic effect of antioxidants on the oxidative stability of soya bean oil- 
and poultry fat-based biodiesel, Journal of American Oil Chemical Society 86 (2009) 459.

doi: https://doi.org/10.1007/s11746-009-1373-8

44. Kinsler, L. E., Rray, A. R., Fundamentals of Acoustics $2^{\text {nd }}$ Ed. Wiley Eastern, New Delhi, India (1989).

45. Ali, A., Hyder, S., Nain A. K., Intermolecular interactions in ternary liquid mixtures by ultrasonic velocity measurements, Journal of Physics 74B (2000) 63.

46. Ernest, S., Kavitha, P., Acoustical and excess thermodynamical parameters of sesame oil in different organic solvents, Journal of Chemical Environmental and Pharmaceutical Research 2 (2011) 4.

47. Kowalski, R., GC analysis of changes in the fatty acid composition of sunflower and olive oils heated with quercetin, caffeic acid, protocatechuic acid, Symposium A Quarterly Journal of Modern Foreign Literatures 18 (2007) 15.

48. Nawar, W. W., Fennema (Ed.) Food Chemistry, $3^{\text {rd }}$ Ed. Marcel Dekker, New York, USA (1996).

49. Vlachos, N., Skopelitis, Y., Psaroudaki, M., Konstantinidou, V., Chatzilazarou, A., Tegou, E., Applications of Fourier transform-infrared spectroscopy to edible oils, Analytica Chimica Acta 574 (2006) 459 doi: https://doi.org/10.1016/j.aca.2006.05.034

50. Innawong, B., Parameswarakumar, M., Marcy, J. E., The determination of frying oil quality using a chemosensory system, Lebensm Wiss Technology 37 (2004) 35. doi: https://doi.org/10.1016/S0023-6438(03)00122-1
51. Kalogianni, E. P., Karapantsios, T. D., Miller, R., Effect of repeated frying on the viscosity, density and dynamic interfacial tension of palm and olive oil, Journal of Food Engineering 105(1) (2011) 169

doi: https://doi.org/10.1016/j.jfoodeng.2011.02.021

52. Farhoosh, R., Hossein, M., Khodaparast, M. H. H., Sharif, A., Rafiee, S. A., Olive oil oxidation: Rejection points in terms of polar, conjugated diene, and carbonyl values, Food Chemistry 131(4) (2012) 1385.

doi: https://doi.org/10.1016/j.foodchem.2011.10.004

53. Frankel, E. N., Huang $S$. W., Improving the oxidative stability of polyunsaturated vegetable oils by blending with high-oleic sunflower oil, Journal of American Oil Chemical Society 71 (1994) 255 doi: https://doi.org/10.1007/BF02638050

54. Romero, A., Cuesta, C., Sanchez, M. F. J., Cyclic fatty acid monomers and thermoxidative alteration compounds formed during frying of frozen foods in extra virgin olive oil, Journal of American Oil Chemical Society 77 (2000) 1169 . doi: https://doi.org/10.1007/s11746-000-0183-5

55. Codex (1995) General Standard for Food Additives (GSFA), Codex STAN Preamble. 192.

56. Rohman, A., Man, Y. B. C., Quantification and classification of corn and sunflower oils as adulterants in olive oil using chemometrics and ftir spectra, The Scientific World Journal 12 (2012) 6 